\title{
EJNSO
}

\section{Endoscopic Versus Microscopic Stapedectomy For Treatment of Otosclerosis}

\author{
Mohamed AM Salem , Hussein Farid Wishahi , Mohammed Azzam Abd El- \\ Raziq, Mina Youssef Asham
}

Department of Otorhinolaryngology, Faculty of Medicine, Assiut University, Assiut, Egypt

\section{Abstract}

Objective: The aim of this study to compare the outcome of endoscopic with that of microscopic stapedectomy.

Methods: This comparative study involved a prospective analysis of patients with conductive hearing loss who underwent stapedectomy at the department of Otorhinolayngology, Assiut University hospital between July 2015 and October 2017. The patients were divided into two groups: Patients in group I were operated with endoscope and patients in group II were operated with microscope. Pure tone audiometry was carried out in all patients preoperatively and postoperatively. Air and bone conduction thresholds were measured at frequencies of 500,1000, 2000 and $4000 \mathrm{HZ}$ and the median and interquartile range (IQ) of the pre and postoperative air-bone gap were noted. Extent of bone work at the posterosuperior part of the external auditory canal, accessibility to the oval window and manipulation of the chorda tympani nerve, postoperative complications and hearing results were also noted and compared between the two groups.

Results: The median preoperative air-bone gap was $35.8 \mathrm{~dB}$ (30.4-45.03) in group I (endoscopic) and $35.85 \mathrm{~dB}$ (32.1-43.38) in group II (microscopic) whereas the median postoperative air-bone gap was $16.7 \mathrm{~dB}$ (5-39.58) in group I and $15.85 \mathrm{~dB}(10-19.58)$ in group II. There was no statistical difference for hearing results between both groups. The need for bone work and manipulation of the chorda tympani nerve for better visualization was more in microscopic group than the endoscopic group and the difference between both groups was statistically significant. The incidence of complications in both groups was nearly the same in both groups and the difference between them was statistically insignificant.

Conclusion: Endoscopic stapedectomy has many advantages over microscopic stapedectomy as better visualization, and easy accessibility to the stapes, oval window niche, and facial nerve. Drilling of the posterosuperior part of the external auditory canal or removal of the scutum and manipulation of the chorda tympani nerve are less frequent with the endoscopic technique.

Keywords: Endoscopic, Microscopic, Stapedectomy, Otosclerosis 


\section{Introduction:}

Stapedectomy consists of removing a portion of the sclerotic stapes footplate and replacing it with an implant that is secured to the incus. This procedure restores continuity of ossicular movement and allows transmission of sound; it depends greatly on the skill and familiarity with the procedure of the surgeon. ${ }^{1}$ Stapedectomies are done in most world centers under microscope with excellent results and low risks. ${ }^{2}$ Since the introduction of stapes surgery by Shea in 1956, numerous modifications of the classical stapes operation have been described in medical literature. ${ }^{3}$ Small hole fenestra technique was defined, different prosthesis models were described, and different instruments were developed to make fenestration. Microscopic surgery of otosclerosis was performed with a great success and minimal complication rates by many authors. ${ }^{4}$ Endoscopes are being used increasingly in middle ear surgery in the last several years. At the beginning endoscopes were used only for diagnostic purposes, but they are now being used more and more for surgery of ear. Many anatomy-based papers have been published, showing the possible advantages of using endoscopes in ear surgery, especially for a better visualization of the structures and recesses within the tympanic cavity. Surgery of otosclerosis seems to be very suitable for exclusively endoscopic approach. However, there are only a few published articles in the literature regarding the endoscopic stapedectomy. 5

\section{Patients and methods:}

This comparative study involved a prospective analysis of patients with conductive hearing loss who underwent stapedectomy at the department of Otorhinolaryngology, Assiut University hospital between July 2015 and October 2017. Local Ethics Committee approval was received. Only stapedectomy patients were included in the study. During our study 40 stapedectomy cases were done and divided into two groups, in group I, 20 cases were operated upon with the endoscope, and in group II, 20 cases were operated upon with the operating microscope but, four cases of the endoscopic stapedectomy group were lost in the postoperative follow up and so, were excluded from the study. Stapedectomy was performed bilaterally in two patients of the microscopic stapedectomy group.

Diagnosis of the patients was based on clinical history and normal otoscopic findings. All patients were evaluated with audiological tests. Patients with airbone gap of $30 \mathrm{~dB}$ or more and with normal otoscopic findings and good cochlear reserve were included in this study. Patients with poor general condition, only hearing ear, poor cochlear reserve, tinnitus and vertigo as essential complaints, active otosclerotic foci as evidenced by a positive Flamingo sign, syndromic conductive deafness, history of ear discharge or trauma and stapedectomy cases lost in the postoperative follow up were excluded from the study. Pure tone audiometry was done by audiologists using mainly Madsen Audiometer, Scotland. Preoperative air-bone gaps of all stapedectomy cases were calculated by subtraction of the average preoperative bone conduction threshold at frequencies 500,1000,2000-4000 HZ from the average of preoperative air conduction threshold of the same mentioned frequencies according to the guidelines of committee of hearing and equilibrium for evaluation of results of treatment of conductive hearing loss, 1995. ${ }^{6}$ Speech audiometry 
tympanometry and acoustic reflex were done preoperatively.

Regarding endoscopic technique it was performed through the permeatal approach under general hypotensive anesthesia. The patients are positioned in the same way as for traditional microscopic otological surgery. The video equipment is placed in front of the surgeon. Epinephrine (1:2000) was injected into the four quadrants of the external auditory canal (EAC). The same instruments and almost the same surgical techniques as in traditional microscopic ear surgery are used with $0^{\circ}, 2.7 \mathrm{~mm}$ diameter and $14 \mathrm{~cm}$ long Karl storz endoscopes. A $4 \mathrm{~mm}$ diameter, $18 \mathrm{~cm}$ long Karl storz endoscope was used when the EAC is wide enough. LED light was mainly used but, Xenon light was used in four cases. The tympanomeatal flap is raised at the wall of the EAC from the 5 o'clock to the 12 o'clock position. The posterior bony part of the EAC was curetted in some cases to better expose the incudostapedial joint and to facilitate the surgical maneuvers Sometimes, Chorda tympani nerve was manipulated for better visualization and inspection of the middle ear, testing of the ossicular mobility was done. After a small test of the ossicular chain immobility, the incudostapedial joint was disarticulated sharply. The stapedial tendon was divided with small curved scissors. The stapes superstructures were down fractured with delicate force and removed, leaving the footplate. Teflon piston prosthesis was sized by measuring the distance from the footplate to the medial surface of the incus. A small hole was created at the midportion or the posterior portion of the footplate with a traditional straight pick instrument. The standard Teflon piston prosthesis, cause fluoroplastic large loop piston " $\mathrm{L}=4.5 \mathrm{~mm}, \mathrm{SD}=0.6$ $\mathrm{mm}, \mathrm{LID}=0.8 \mathrm{~mm} "$ is placed between the oval window and the incus. The malleus is carefully palpated to ensure movement of the ossicles all the way through the prosthesis. The tympanomeatal flap was repositioned and sealed with Gelfoam treated with antibiotic solution. All patients received antibiotics for 2 weeks, injectable ceftriaxone for 3 days then amoxicillinclavulanic acid tablets for the rest of two weeks if there was allergy to penicillin the patients received fluoroquinolones, a short steroid taper, and pain medications post-operatively. Patients were counseled about feeling dizzy postoperatively. When they are able to tolerate an oral meal, they are discharged home and scheduled for follow up two weeks later. At that time, we removed any remaining packing from the external auditory canal.

Regarding microscopic stapedectomy surgery was done through the permeatal approach in all cases under general anesthesia with orotracheal intubation and hypotensive technique using Leica M 320 HD microscope, Switzerland.

The postoperative air-bone gaps of all stapedectomy cases was assessed by PTA at 2 to 6 months after the surgery by subtraction of the average of preoperative bone conduction threshold at frequencies 500, 1000, 2000-4000 HZ from the average of postoperative air conduction threshold at the same mentioned frequencies according to the guidelines of committee of hearing and equilibrium for evaluation of results of treatment of conductive hearing loss, 1995. ${ }^{6}$ Hearing gain which is the difference in the preoperative and postoperative air-bone gap was also assessed. The parameters that were investigated for comparison between the two techniques were Intraoperative complications, extent of bone work at the posterosuperior part of the external auditory canal, accessibility to the oval window and manipulation of the chorda tympani nerve and postoperative hearing gain, the average of postoperative air- 
bone gap, postoperative dizziness and postoperative complications as facial nerve palsy and taste changes.

\section{Statistical analysis:}

Numerical data were explored for normality by checking the distribution of data and using tests of normality (Kolmogorov-Smirnov and ShapiroWilk tests). Age data showed normal (parametric) distribution while bone loss data showed non-normal (nonparametric) distribution. Data were presented as mean, median, standard deviation (SD) and range values. For parametric data; Student's t-test was used to compare between the two groups. For non-parametric data; Mann-Whitney $U$ test was used to compare between the two groups. Qualitative data were presented as frequencies and percentages. Chisquare test (or Fisher's Exact test when applicable) were used for comparisons regarding qualitative data. The significance level was set at $\mathrm{P} \leq 0.05$. Statistical analysis was performed with IBM SPSS Statistics Version 20 for Windows.

\section{$\underline{\text { Results: }}$}

Tables 1, 2, 3 show the demographic data of the study. During our study 40 stapedectomy cases were done and were divided into two groups, in group I, 20 cases were operated upon with the endoscope but, four cases were lost in the postoperative follow up so, were excluded from the study, in group II, 20 cases were operated upon with the operating microscope. This study included 36 ears, 34 patients '14 male and 20 female patients' (as in microscopic group two patients underwent the surgery in both ears so, the microscopic group included 18 patients and the endoscopic group included 16 patients).

\begin{tabular}{|c|c|c|c|c|}
\hline \multirow{2}{*}{ Gender } & \multicolumn{2}{|c|}{ Group I } & \multicolumn{2}{c|}{ Group II } \\
\cline { 2 - 5 } & \multicolumn{2}{|c|}{$\begin{array}{c}\text { Endoscopic } \\
\text { stapedectomy }\end{array}$} & \multicolumn{2}{c|}{$\begin{array}{c}\text { Microscopic } \\
\text { stapedectomy }\end{array}$} \\
\hline & $\begin{array}{c}\mathrm{n}= \\
16\end{array}$ & $\%$ & $\mathrm{n}=18$ & $\%$ \\
\hline Male & 5 & 31.25 & 9 & 50.0 \\
\hline Female & 11 & 68.75 & 9 & 50.0 \\
\hline
\end{tabular}

Table 1: Gender in Endoscopic and microscopic stapedectomy

\begin{tabular}{|c|c|c|c|c|}
\hline \multirow{3}{*}{ Age range } & \multicolumn{2}{|c|}{ Group I } & \multicolumn{2}{|c|}{ Group II } \\
\hline & \multicolumn{2}{|c|}{$\begin{array}{l}\text { Endoscopic } \\
\text { stapedectomy }\end{array}$} & \multicolumn{2}{|c|}{$\begin{array}{l}\text { Microscopic } \\
\text { stapedectomy }\end{array}$} \\
\hline & $n=16$ & $\%$ & $n=18$ & $\%$ \\
\hline $\begin{array}{l}\text { Median } \\
\text { (IQ) }\end{array}$ & \multicolumn{2}{|c|}{$32(25.25-36.75)$} & \multicolumn{2}{|c|}{$34(27.5-40)$} \\
\hline
\end{tabular}

Table 2: Age range in Endoscopic and microscopic stapedectomy

\begin{tabular}{|c|c|c|c|c|}
\hline \multirow{2}{*}{ Side } & Group I & \multicolumn{2}{|c|}{ Group II } \\
\cline { 2 - 5 } & $\begin{array}{c}\text { Endoscopic } \\
\text { stapedectomy } \\
(\mathbf{n}=16)\end{array}$ & $\begin{array}{c}\text { Microscopic } \\
\text { stapedectomy } \\
(\mathbf{n}=\mathbf{2 0})\end{array}$ \\
\hline & $\mathrm{n}$ & $\%$ & $\mathrm{n}$ & $\%$ \\
\hline $\begin{array}{c}\text { Left } \\
\text { Stapedectomy }\end{array}$ & 8 & $\mathbf{5 0 . 0}$ & 12 & $\mathbf{6 0 . 0}$ \\
\hline $\begin{array}{c}\text { Right } \\
\text { stapedectomy }\end{array}$ & 8 & $\mathbf{5 0 . 0}$ & 8 & $\mathbf{4 0 . 0}$ \\
\hline
\end{tabular}

Table 3: Side in Endoscopic stapedectomy and Microscopic stapedectomy

Stapedectomy was performed on the right ear in 16 cases and the left ear in 20 cases. Median age was 32 years (25.25 - 36.75) in the endoscopic group and 34 years $(27.5$ - 40) in the microscopic group. The median preoperative air-bone gap was $35.8 \mathrm{~dB}$ (30.4-45.03) in group I (endoscopic) and $35.85 \mathrm{~dB}(32.1-43.38)$ in group II (microscopic) whereas the median postoperative air-bone gap was 16.7 $\mathrm{dB}(5-39.58)$ in group $\mathrm{I}$ and $15.85 \mathrm{~dB}$ (10-19.58) in group II as shown in table 4. There was no statistical difference for 
hearing results between both groups as shown in table 4 . Bone work in the form of curetting of posterior scutum for better accessibility to the oval window was done in 3 cases of the endoscopic group and 12 cases of the microscopic group and the difference was statistically significant $(\mathrm{P}=0.013)$ as shown in table 5.

Chorda tympani nerve was manipulated in some cases for better visualization of the oval window area and stapes footplate it was manipulated in 3 cases of endoscopic stapedectomy while in microscopic stapedectomy it was manipulated in 12 cases so; the difference was statistically significant $(P$ $=0.031)$ as shown in table 6 . The difference in complications between the two groups was statistically insignificant $(\mathrm{p}=0.492)$ as shown in table 7 . One patient of the microscopic group developed delayed partial right lower motor neuron (LMN) facial nerve paralysis but, patient was first seen 3 weeks postoperatively and improved by medical treatment completely. In group I, on postoperative follow up audiograms four patients (25\%) developed mixed hearing loss and considered as failed cases. In group II, on postoperative follow up audiograms one patient $(5 \%)$ developed mixed hearing loss, another one patient $(5 \%)$ developed SNHL and one patient (5\%) developed moderate CHL they were considered as failed case. Two patients of group II developed TM perforation postoperatively discovered on follow up visits one of them showed spontaneous healing, the other one was totally satisfied of hearing improvement and not complained of otorrhea she was planned for tympanoplasty but, she refused. Only one patient of group I, developed TM perforation but, spontaneous healing was noticed on follow up 2 months postoperatively. In spite of the manipulation of the Chorda tympani nerve in 12 cases of group II, only one patient complained of taste abnormalities but, this was insignificant and improved in postoperative follow up 6 months later, none of the 3 patients of group I, with manipulated Chorda tympani complained of taste abnormalities. Postoperative dizziness was observed in eight cases of each group I and in ten cases of group II, but the difference was not statistically significant $(\mathrm{P}=0.858)$ as shown in table 8. 


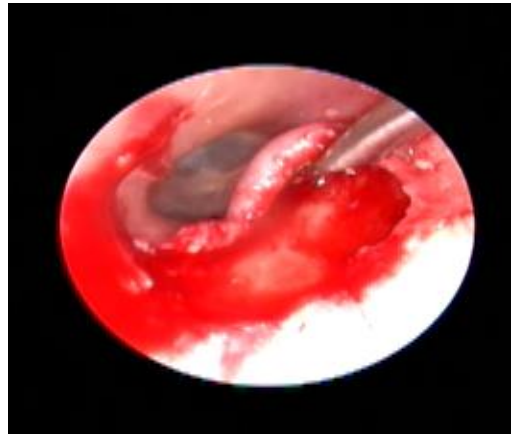

A.Elevation of the left tympanomeatal flap

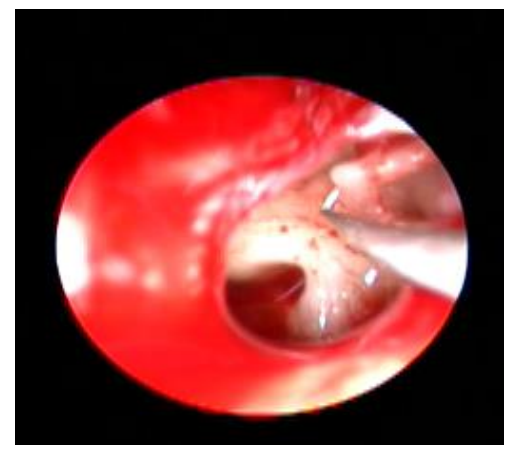

C.Separation of the left incudostapedial joint

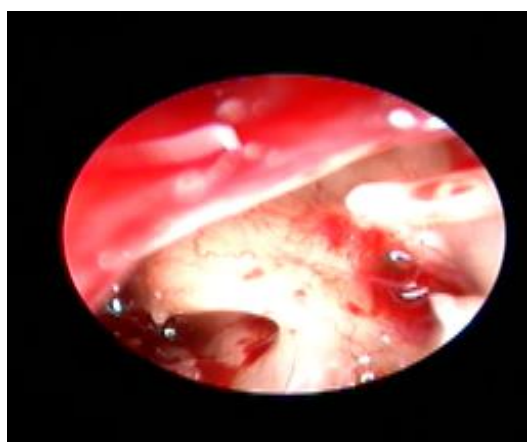

E. Creation of left stapedotomy

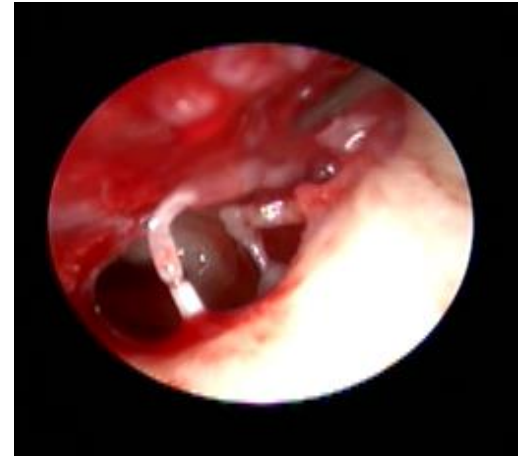

B.Endoscopic view of the left middle ear

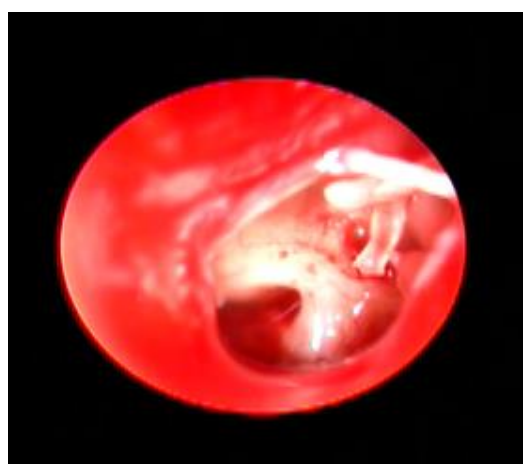

D.Removal of the left stapes superstructures

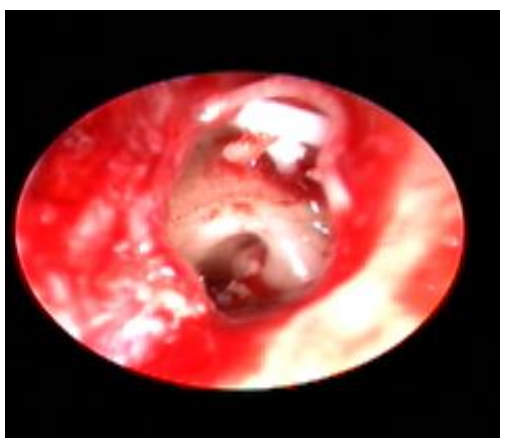

F.Application of the prosthesis in the left ear

Fig. 1. Shows the steps of endoscopic stapedectomy 
Fig 2 shows some steps of microscopic stapedectomy

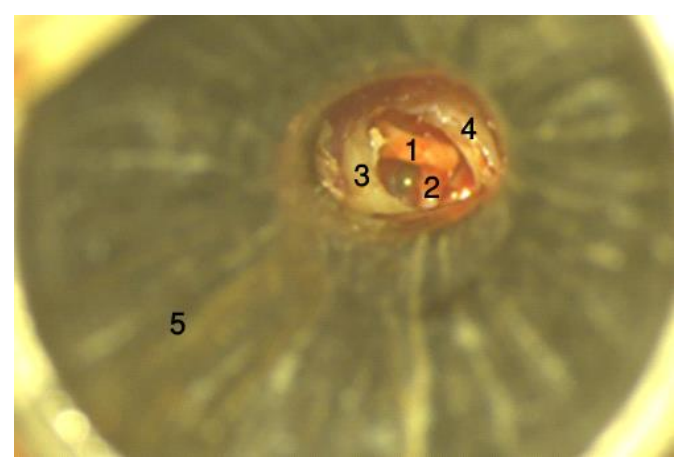

-Incus long process

2-Stapes

3- Posterior scutum

4- Chorda tympani nerve after

displacement

5- Speculum

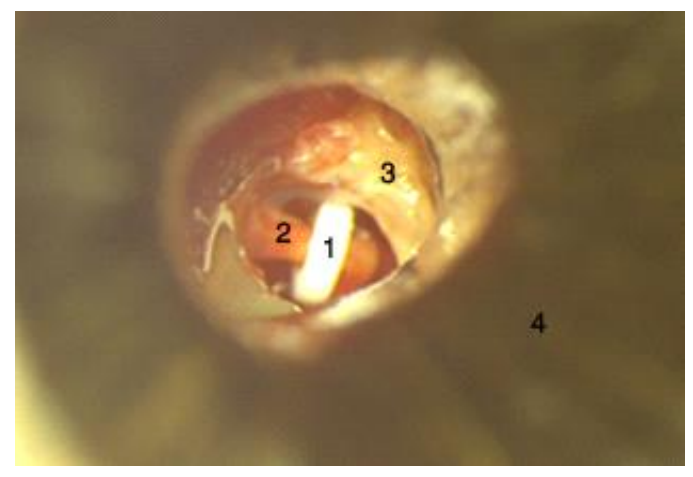

1-Teflon piston prosthesis

2-Incus long process

3-Chorda tympani nerve

4-Speculum

Table 4: Preoperative and Postoperative air-bone gap in Endoscopic stapedectomy and Microscopic stapedectomy

\begin{tabular}{|c|c|c|c|c|c|}
\hline \multirow{2}{*}{ Air-bone gap (ABG) } & Group I & Group II & \multirow{2}{*}{ Z } & \multirow{2}{*}{ P1 } \\
\cline { 2 - 4 } & Endoscopic & Microscopic & & \\
\hline Pre-operative & $\mathbf{3 5 . 8 ( 3 0 . 4 - 4 5 . 0 3 )}$ & $\mathbf{3 5 . 8 5}(32.1-43.38)$ & -0.256 & 0.798 \\
\hline Median (IQ range) & \multicolumn{5}{|l}{} \\
\hline Post-operative & $16.7(5-39.58)$ & $15.85(10-19.58)$ & -0.384 & 0.701 \\
\hline Median (IQ range) & -2.561 & -3.924 & & \\
\hline Z & $0.010 *$ & $0.000 * *$ & & \\
\hline P2 & & & & \\
\hline
\end{tabular}

P1: Comparison between the two groups in pre-operative and post-operative using Mann-Whitney $\mathbf{U}$ test. P2: Comparison between pre-operative and post-operative in each group using Wilcxon test.

* Statistically significant difference $(\mathrm{p}<0.05)$, ** Highly statistically significant difference $(\mathrm{p}<0.01)$. 
Table 5: Bone work in Endoscopic stapedectomy and Microscopic stapedectomy

\begin{tabular}{|c|c|c|c|c|c|}
\hline \multirow{3}{*}{ Bone work } & \multirow{2}{*}{\multicolumn{2}{|c|}{$\frac{\text { Group I }}{\text { Endoscopic }}$}} & \multirow{2}{*}{\multicolumn{2}{|c|}{$\frac{\text { Group II }}{\text { Microscopic }}$}} & \multirow{3}{*}{$\begin{array}{c}P . \\
\text { value }\end{array}$} \\
\hline & & & & & \\
\hline & No. & $\%$ & No. & $\%$ & \\
\hline Needed & 3 & 18.75 & 12 & 60.0 & \multirow{2}{*}{$0.013 *$} \\
\hline Not needed & 13 & 81.25 & 8 & 40.0 & \\
\hline
\end{tabular}

Table 6 : Manipulation of the chorda tympani nerve in Endoscopic stapedectomy and Microscopic stapedectomy

\begin{tabular}{|c|c|c|c|c|c|}
\hline \multirow{2}{*}{$\begin{array}{c}\text { Manipulation and injury of the } \\
\text { chorda tympani nerve }\end{array}$} & \multicolumn{2}{|c|}{ Group I } & \multicolumn{2}{|c|}{ Group II } & \\
\cline { 2 - 6 } & \multicolumn{2}{|c|}{$\begin{array}{c}\text { Endoscopic } \\
\text { stapedectomy }\end{array}$} & \multicolumn{2}{c|}{$\begin{array}{c}\text { Microscopic } \\
\text { stapedectomy }\end{array}$} & \\
\cline { 2 - 7 } & $\mathrm{n}=16$ & $\%$ & $\mathbf{n = 2 0}$ & $\%$ & P. value \\
\hline No & 13 & 81.75 & 8 & 40 & \multirow{2}{*}{$0.031 * *$} \\
\hline Yes & 3 & 18.25 & 12 & 60 & \\
\hline
\end{tabular}

Table 7: Postoperative Complication in Endoscopic stapedectomy and Microscopic Stapedectomy

\begin{tabular}{|c|c|c|c|c|c|}
\hline \multirow{3}{*}{$\begin{array}{l}\text { Postoperative } \\
\text { Complications }\end{array}$} & \multicolumn{2}{|c|}{ Group I } & \multicolumn{2}{|c|}{ Group II } & \multirow{3}{*}{ P. value } \\
\hline & \multicolumn{2}{|c|}{$\begin{array}{c}\text { Endoscopic } \\
\text { stapedectomy }\end{array}$} & \multicolumn{2}{|c|}{$\begin{array}{c}\text { Microscopic } \\
\text { stapedectomy }\end{array}$} & \\
\hline & $n=16$ & $\%$ & $\mathbf{n}=\mathbf{2 0}$ & $\%$ & \\
\hline No & 11 & 68.7 & 13 & 65 & \multirow{8}{*}{0.492} \\
\hline Yes & 5 & 31.3 & 7 & 35 & \\
\hline Facial palsy & $\mathbf{0}$ & 0.0 & 1 & 5.0 & \\
\hline Mixed HL & 4 & 25.0 & 1 & 5.0 & \\
\hline SNHL & $\mathbf{0}$ & 0.0 & 1 & 5.0 & \\
\hline CHL & $\mathbf{0}$ & $\mathbf{0}$ & 1 & 5.0 & \\
\hline Taste changes & $\mathbf{0}$ & 0.0 & 1 & 5.0 & \\
\hline TM perforation & 1 & 6.3 & 2 & 10.0 & \\
\hline
\end{tabular}

Table 8: Postoperative dizziness in Endoscopic stapedectomy and Microscopic stapedectomy

\begin{tabular}{|l|c|c|c|c|c|}
\hline \multirow{2}{*}{ Postoperative Dizziness } & \multicolumn{2}{|c|}{ Group I } & \multicolumn{2}{|c|}{ Group II } & \multirow{2}{*}{$\begin{array}{c}\text { P. } \\
\text { value }\end{array}$} \\
\cline { 2 - 6 } & \multicolumn{2}{|c|}{ Endoscopic } & \multicolumn{2}{|c|}{ Microscopic } & \\
\cline { 2 - 6 } & No. & $\%$ & No. & $\%$ & \\
\hline
\end{tabular}




\section{Discussion :}

The number of reports about endoscopic ear surgery shows marked increase in recent years. Endoscopic ear surgery is suitable for ossiculoplasty, otosclerosis, and chronic otitis media. ${ }^{7}$

Poe in 2000 described endoscope assisted stapedectomy. ${ }^{3}$ A study by Kojima et al 2014 compared 15 cases of endoscopic stapedectomy to 41 cases of microscopic stapedecomy regarding postoperative hearing, complications and extent of drilling at the posterosuperior part of the EAC reported that stapes surgery can be performed with an endoscope despite the presence of a curved and narrow EAC and is minimally invasive because less bone work was required and there were fewer chorda tympani injuries. ${ }^{8}$ In that study, chorda tympani injury and the need for bone work in the posterosuperior part of the ECA were very low in the endoscopic stapedectomy group in comparison to the microscopic stapedectomy group which is similar to our study.

Endoscopic stapedectomy has the advantage of good visualization, and easy accessibility to the stapes, oval window niche and facial nerve 9 which agrees with our study as evidenced by the less need for bone work in the posterosuperior part of the external auditory canal in the endoscopic group than the microscopic group. Kojima et al 2014 reported that damage of the chorda tympani usually occurred in the classic microscopic stapedectomy due to its manipulation as more of the posterior part of the external auditory canal should be removed in trial for optimal visualization of the oval window which is in agreement with our study it was not injured at all in endoscopic stapedectomy cases. ${ }^{8}$ Some literatures reported that experience is required for fully endoscopic stapes surgery ${ }^{8,9}$ which agreed with our study as evidenced by the previous result of postoperative airbone gap in endoscopic stapedectomy. A study by Naik and Nemade,2014 on endoscopic stapedectomy had not reported any complications as taste failure, facial nerve paralysis and tympanic membrane perforation. ${ }^{10}$ In our study, in agreement with Naik and Nemade 2014, our study on endoscopic assisted stapedectomy did not report any patient with taste failure and facial nerve paralysis but, in contrast to Naik and Nemade, 2014 it reported one case of postoperative tympanic membrane perforation. In a study by Surmeliglu et al 2016 twenty two cases of endoscopic stapedectomy were performed and compared to 24 cases of microscopic stapedectomy regarding hearing results and complications, the median postoperative air-bone gap was $6.6 \mathrm{~dB}$ (0-30) in the endoscopic group and 10 $\mathrm{dB}(1.6-35)$ in the microscopic group and there was no statistically significant difference between the two groups. ${ }^{9}$ In our study the median postoperative airbone gap was $16.7 \mathrm{~dB}(5-39.58)$ in the endoscopic group and was $15.85 \mathrm{~dB}$ (1019.58) in the microscopic group and there was no statistically significant difference between the two groups which is in agreement with Surmeliglu et al 2016. In another study, Nogueira et al 2011 showed that fully endoscopic stapes surgery is technically feasible, safe and promising. In their series of 15 patients, the main advantages were virtually no trauma to the chorda tympani nerve and excellent vision. The disadvantages were the lack of stereoscopic vision, having to work with only one hand, and the learning curve. ${ }^{11}$ Regarding postoperative complications Kojima et al 2014 reported that one patient of the endoscopic group developed late facial paralysis (HouseBrackmann grade 1). ${ }^{8}$

In contrast to our study no patient of the endoscopic group developed facial 
nerve. In contrast to our study no patient of the endoscopic group developed facial nerve paralysis but, one patient of the microscopic group developed postoperative late facial paralysis (House-Brackmann grade 2). In agreement with Kojima et al 2014 none of patients in our study in the endoscopic group developed postoperative impaired taste sensation whereas in our study there was one patient of the microscopic group developed postoperative impaired taste sensation, in Kojima et al 2014 study 4 patients of microscopic group developed taste abnormalities. ${ }^{8}$ In Surmeliglu et al in 2016 the incidence of chorda tympani nerve injury was more in the microscopic group than the endoscopic group ${ }^{9}$ which is in agreement with our study but, impaired taste sensation was noticed in $33 \%$ in the microscopic group and in $4.5 \%$ of the endoscopic group ${ }^{8}$ which is in contrast to our study as only one patient of the microscopic group developed impaired taste sensation and no patients of the endoscopic group developed impaired taste sensation but, this improved in postoperative follow up 6 months later. Our study was in agreement with Surmeliglu et al 2016 regarding the incidence of tympanic membrane perforation as two cases $(10 \%)$ of the microscopic group developed TM perforation and one case (6.3\%) of the endoscopic group developed TM perforation ${ }^{9}$. Regarding postoperative hearing loss and tinnitus, in contrast to Surmeliglu et al 2016 as none of his patients in the study of both groups developed sensorineural or mixed hearing loss ${ }^{9}$; in our study 4 patients of the endoscopic group developed mixed HL postoperatively and were considered as failed cases. One patient of microscopic developed mixed HL and tinnitus, one patient developed SNHL; another one developed moderate CHL and were considered as failed cases. the increased number of cases of endoscopic surgery developed mixed HL and nerve affection postoperatively maybe due to the damaging thermal effect of the xenon light that was used with the 0-degree rigid endoscope with an outer diameter of $4 \mathrm{~mm}$ during our surgery which could be transmitted easily from the tip of the endoscope to the oval window then through the perilymph to the cochlea.

A study by Nogueira et al 2011 reported that the tips of nasal endoscopes tend to directly transfer the heat generated by the light sources, it may have a high temperature which may damage some structures. ${ }^{11}$ Dundar et al 2015 reported that endoscopes can cause high temperature in the middle ear and inner ear through an animal study showed that halogen and xenon light caused a temperature increase in the oval window which in turn could easily pass to the cochlea by perilymph and could cause sensorineural hearing loss. 12 Also, Ito et al 2016 reported that the use of 2.7-mm endoscope coupled to an LED light source is safe in ear surgery, but operators should not exceed clinical settings particularly with 4-mm endoscope with a xenon light source to avoid tissue injury. ${ }^{13}$ Tomazic et al 2014 measured the light intensity and temperature at the tip of 4-mm endoscope during nasal sinus surgery using three types of light sources: LED, xenon and halogen and found that LED is the safest and provide very good illumination; whereas xenon has the greatest illumination, but generates potential tissue damaging temperature ${ }^{14}$.

\section{Conclusion:}

Endoscopic stapedectomy has many advantages over microscopic stapedectomy as better visualization, and easy accessibility to the stapes, oval window niche, and facial nerve. Drilling of the posterosuperior part of the external auditory canal or removal of the 
scutum and manipulation of the chorda tympani nerve are less frequent with the endoscopic technique. The postoperative hearing results are nearly the same in both techniques. Postoperative complications such as chorda tympani injury and resultant taste problems and facial nerve paralysis were less in endoscopic than microscopic stapedectomy. Education of surgical assistants is better with endoscopic stapedectomy due to better visualization. On the other hand, endoscopic stapes surgery has some limitations such as one-handed surgery, experience is required, and it lacks the stereoscopic vision.

\section{Financial disclosure}

None.

\section{Conflict of interest}

None declared.

\section{Reference:}

1. De Souza C.,Glasscock, M.E.(2003): Otosclerosis and Stapedectomy : Diagnosis and Management of Complications . New York, Thieme; 1 st edition.

2. Tarabichi M. (1999): Endoscopic middle ear surgery. Ann Otol Rhinol Laryngol; 108:39-46.

3. Poe DS. (2000): Laser assisted endoscopic stapedectomy: a prospective study.Laygoscope; 110:1-37.

4. Fisch U. (1982): Stapedotomy versus stapedectomy. American Journal of Otology; 4:112-117.

5. Sarkar S, Banerjee S, Chakravarty S, Singh R, Sikder B, Bera SP. (2013) Endoscopic stapes surgery: our experience in thirty two patients. Clin.Otolaryngol; 270:1547-9.

6. Committee on Hearing and Equilibrium guidelines for the evaluation of results of treatment of conductive hearing loss. American Academy of Otolaryngology-Head and Neck Surgery Foundation, Inc. Otolaryngol Head Neck Surg 1995;113:186-187.

7. Migirov L, Shapira Y, Horowitz Z. (2011): Exclusive endoscopic ear surgery for acquired cholesteatoma. Otol Neurotol; 32:433-436.

8. Kojima H, Kornori M, Chikazawa $\mathrm{S}$, Yaguchi $\mathrm{Y}$, Yamamoto $\mathrm{K}$, Chujo K, et al. (2014): Comparison between endoscopic and microscopic stapes surgery. Laryngoscope; 124:266-71.

9. Surmelioglu Ozgur, Suleyman Ozdemir, Ozgur Tarkan, UlkuTuncer, Muhammed Dagkiran, Fikret Cetik. (2016): Endoscopic versus microscopic stapes surgery, Auris Nasus Larynx; 07:001.

10. Naik C, Nemade S. (2014): Endoscopic stapedotomy: our view point. Eur Arch Otorhinolaryngol.

11. Nogueira Junior JF, Martins MJ, Aguiar CV, Pinheiro Al. (2011): Fully endoscopic stapes surgery (stapedotomy): technique and preliminary results. Braz J Otorhinolaryngol; 77:721-7.

12. Dundar R, Bulut H, Guler OK, Yukkaldiran A, Demirtas Y, lynen I, Ferhat Bozkus, Erkan Kulduk. (2015): Oval Window Temperature Changes in an Endoscopic Stapedectomy, Journal of Craniofacial Surgery; 26:17048.

13. Ito $\mathrm{T}$, Kubota $\mathrm{T}$, Takagi $\mathrm{A}$, Watanabe T, Futai K, Furukawa T, Kakehata S. (2016): Safety of heat generated by endoscope light sources in simulated transcanal endoscopic ear surgery / Auris Nasus Larynx; 43:501-506. 
14. Tomazic PV, Hammer GP, Gerstenberger C, Koele W, Stammberger H. (2012): Heat development at nasal endoscope tips: danger of tissue damage? A laboratory studies. Laryngoscope; 122:1670-3 